\title{
Lista das espécies de Hippoboscoidea (Diptera) no estado de Mato Grosso do Sul, Brasil
}

\author{
Gustavo Graciolli', Luiz Felipe A. C. Carvalho', Alan F. Eriksson', \\ Camila de Lima Silva' \& Daniel M. C. Alcântara²
}

\footnotetext{
1. Centro de Ciências Biológicas e da Saúde, Universidade Federal de Mato Grosso do Sul, Caixa Postal 549, 79070-900, Campo Grande, Mato Grosso do Sul, Brasil. (ggraciolli@yahoo.com.br)

2. Departamento de Zoologia, Instituto de Biociências, Universidade de São Paulo. Rua do Matão, Travessa 14, nº 101 Cidade Universitária, 05508-900, São Paulo, São Paulo, Brasil.
}

Recebido 18 novembro 2016

Aceito 6 fevereiro 2017

DOI: $10.1590 / 1678-4766 e 2017137$

ABSTRACT. Checklist of Hippoboscoidea (Diptera) of the state of Mato Grosso do Sul, Brazil. A checklist of the species of Hippoboscidae, Nycteribiidae and Streblidae and their hosts and localities in the state of Mato Grosso do Sul, based on literature and specimens deposited in scientific collections, is presented. Fifty-three species are recorded, being Xenotrichobius noctilionis Wenzel, 1976 reported for first time in Brazil.

KEYWORDS. Biota-MS Program, Cerrado, Pantanal, Floresta Atlântica, host.

RESUMO. Uma listagem das espécies de Hippoboscidae, Nycteribiidae e Streblidae, seus hospedeiros e localidades no estado de Mato Grosso do Sul, baseada em dados de literatura e no acervo de coleções científicas, é apresentada. Cinquenta e três espécies são registradas, sendo Xenotrichobius noctilionis Wenzel, 1976 reportada pela primeira vez no Brasil.

PALAVRAS-CHAVE. Programa Biota-MS, Cerrado, Pantanal, Floresta Atlântica, hospedeiro.

Na superfamília Hippoboscoidea estão arroladas quatro famílias de moscas caliptradas, Glossinidae, Hippoboscidae, Nycteribiidae e Streblidae (McAlPInE, 1989). Glossinidae compreende moscas hematófagas restritas à Região Etiópica, enquanto as outras três famílias incluem moscas hematófagas, ectoparasitas de aves e mamíferos e com distribuição cosmopolita. Hippoboscidae são moscas ectoparasitas de aves e mamíferos (artiodáctilos, lêmures e cangurus) (MAA, 1969). No continente americano foram registradas 49 espécies de 12 gêneros e no Brasil 30 espécies de 10 gêneros (MAA, 1969). Nycteribiidae e Streblidae são formadas por espécies exclusivamente ectoparasitas de morcegos (Dick \& Miller, 2010; Graciolli, 2010). No continente americano foram registradas dois gêneros e 53 espécies de nicteribiídeos (Graciolli, 2010) e 27 gêneros e 159 espécies de estreblídeos (Dick \& GRACIOLLI, 2008; Graciolli \& Azevedo, 2011; Graciolli \& Dick, 2012; PoInar \& Brown, 2012), sendo um gênero e espécie fóssil (PoInar \& Brown, 2012). No Brasil, são conhecidas até o momento dois gêneros e 26 espécies (Graciolli et al., 2007) e 20 gêneros e 74 espécies (Aguiar et al., 2006; ERIKSSON et al., 2011; Graciolli et al., 2008, 2010; GraCiOLLI \& AzEVEDO, 2011; GRACIOLLI \& Dick, 2012) de nicteribiídeos e estreblídeos, respectivamente.

Nesta contribuição é apresentada uma listagem das espécies das famílias Hippoboscidae, Nycteribiidae e Streblidae no Estado de Mato Grosso do Sul.

\section{MATERIAL E MÉTODOS}

A listagem foi feita a partir de dados de literatura e de espécimes depositados na Coleção Zoológicas de Referência da Universidade Federal de Mato Grosso do Sul (ZUFMS) e na Coleção Entomológica da Universidade Para o Desenvolvimento do Estado de Mato Grosso do Sul (Uniderp/Anhanguera), ambas em Campo Grande. $\mathrm{Na}$ listagem as famílias, assim como subfamílias, gêneros e espécies em cada família são apresentadas em ordem alfabética. Para cada espécie as seguintes informações são apresentadas: espécie hospedeira, município e a localidade onde o díptero hipoboscoídeo foi coletado sobre a espécie hospedeira e a fonte da informação (referência bibliográfica e/ou coleção científica). A espécie hospedeira ou a localidade precisa no estado pode estar ausente. Os nomes científicos dos hospedeiros estão apresentados em ordem alfabética. As localidades, identificadas por algarismos, estão representadas em um mapa (Fig. 1).

Em relação aos hospedeiros, os nomes subespecíficos não foram utilizados. Para os nomes válidos e sinonímias das espécies de aves seguiu-se a Lista das Aves do Brasil, 


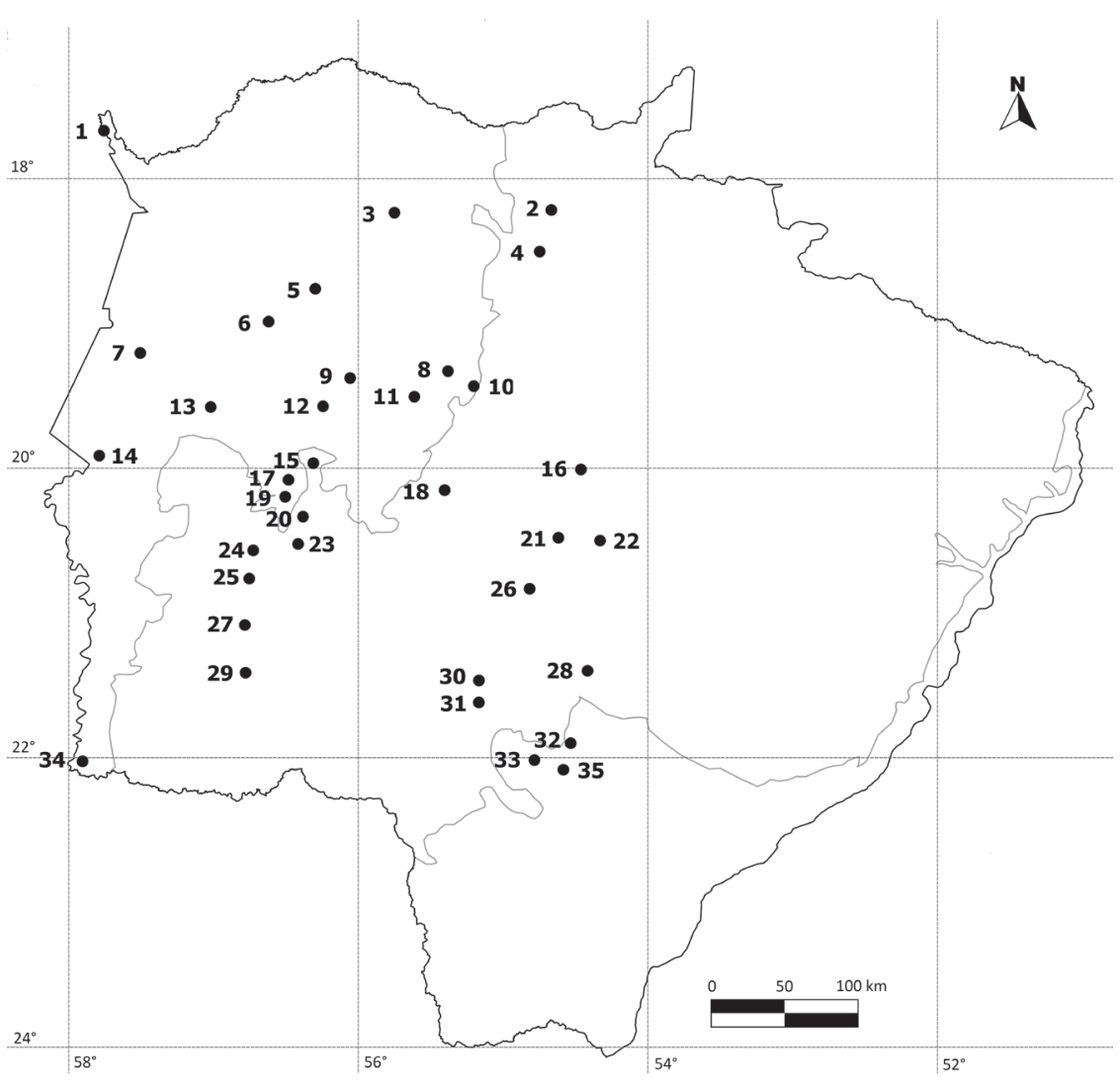

Fig. 1. Localidades, com coordenadas geográficas, onde foram coletados espécimes de Hippoboscoidea no Estado do Mato Grosso do Sul, Brasil: 1, Porto

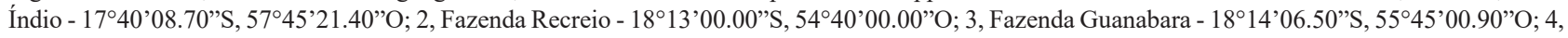

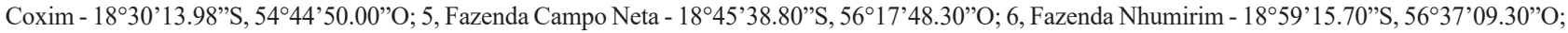

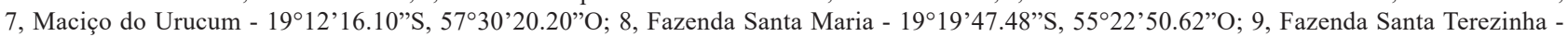

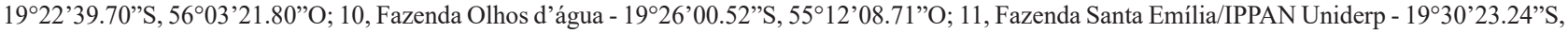
$55^{\circ} 36^{\prime} 46.10^{\prime \prime} \mathrm{O} ; 12$, Fazenda Rio Negro - 19³4’22.00”S, 56¹4’36.00”O; 13, Fazenda São Bento/Base de Estudos do Pantanal UFMS - 19³4’38.90”S,

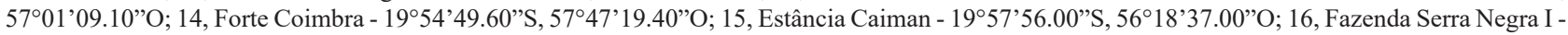

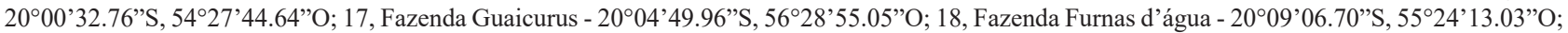
19, Salobra - 20¹1'52.00”S, 56³0'17.00”O; 20, Fazenda São Cristóvão/Miranda - 20²0’06.29”'S, 56²2’53.90”O; 21, Campo Grande - 20²8'51.43”S,

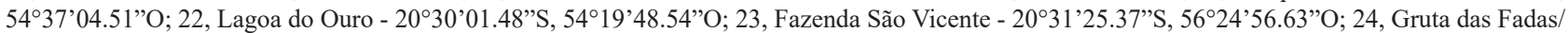
Bodoquena - 20³4’04.76”S, 5643'32.02”O; 25, Gruta “Seu” Natal e Complexo de Grutas do Córrego Azul - 2045'46.96”'S, 5645'10.02”O; 26,

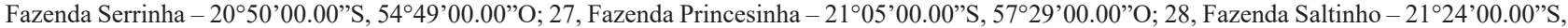

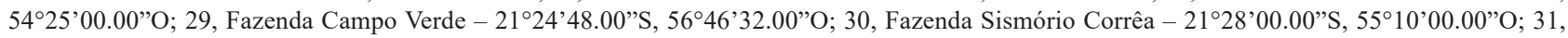

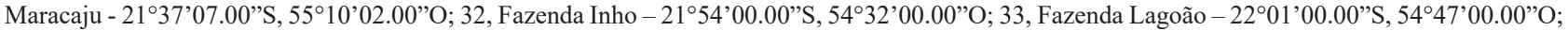

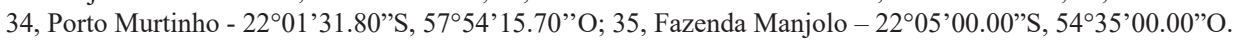

instituída pelo Comitê Brasileiro de Registros Ornitológicos (CBRO, 2011), para os de cervídeos TIEPOLO \& TOMAs (2011) e para os de morcegos GARDNER (2007), com exceção para as espécies de Natalus Gray, 1838 para as quais adotou-se GARBINO \& TEJEDOR (2013).

\section{RESULTADOS E DISCUSSÃO}

Foram encontradas 11 espécies de Hippoboscidae, três de Nycteribiidae e 39 de Streblidae, totalizando 53 espécies. Xenotrichobius noctilionis é registrada pela primeira vez no Brasil, aumentando para 21 e 75 o número de gêneros e espécies, respectivamente, de estreblídeos registrados em território brasileiro.

\section{Lista das espécies de Hippoboscoidea e de seus hospedeiros para o estado de Mato Grosso do Sul.}

\section{HIPPOBOSCIDAE}

\section{Lipopteninae}

1. Lipoptena guimaraesi Bequaert, 1957. Ozotoceros bezoarticus (Linnaeus, 1758), Corumbá (6) (GraCiOLLI et al., 2011).

2. Lipoptena mazamae (Rondani, 1878). Ozotoceros bezoarticus, Corumbá (6) (Graciolli et al., 2011). Hospedeiro não determinado, Miranda (19) (BEQUAERT, 1957); espécimen volante capturado em armadilha Malaise, Campo Grande (21) (ZUFMS).

Ornithomyinae 
3. Icosta albipennis (Say, 1823). Eurypyga helias (Pallas, 1781), "Rio Paraná" (Bequaert, 1955).

4. Icosta nigra (Perty, 1833). Accipiter bicolor pileatus (Temminck, 1823), Coxim (2) (Bequaert, 1955). Caracara plancus (Miller, 1777), Bodoquena (24) BeQUAert, 1955). Cathartes aura (Linnaeus, 1758), Maracaju (31) (BEQuAert, 1955). "fulvous-bellied kite", Maracaju (31) (BeQuaert, 1955). Gampsonyx swainsonii Vigors, 1825, Miranda (19) (BeQuaERT, 1955). Heterospizias meridionalis (Latham, 1790), Coxim (2) (Bequaert, 1955). Comentário. A identificação na língua inglesa "Fulvous-bellied Kite" em BEQUAERT (1955) não é um nome reconhecido entre os ornitólogos e provavelmente este denominação tratase de uma descrição rápida da ave feita pelo coletor de I. nigra em Maracaju. "Kites" são denominados em gaviões (Accipitridae) de pequeno tamanho corporal (às vezes de médio) e "fulvous-belly" seria alguma ave apresentado uma "barriga amarronzada". Os hospedeiros de I. nigra em Maracaju poderiam ser Leptodon cayanensis (Latham, 1790) e Chondrohierax uncinatus (Temminck, 1822), considerando as espécies de gaviões morfologia e/ou fases de plumagem que poderiam ser caracterizado por apresentar um ventre amarronzado e que ocorrem na região de Maracaju (Fernando Costa Straube, Hori Consultoria Ambiental, com. pessoal).

5. Microlynchia crypturelli Bequaert, 1938. Crypturellus parvirostris (Wagler, 1827), Coxim (2) (BEQUAERT, 1955).

6. Microlynchia pusilla (Speiser, 1902). Crypturellus sp., Maracaju (31) (BEQUaERT, 1955).

7. Olfersia bisulcata Macquart, 1847. Cathartes aura, Maracaju (31) (BEQUaERT, 1957).

8. Olfersia coriacea Wulp, 1903. Crax sp. Maracaju (31) (Bequaert, 1957). Mesembrinibis cayennensis (Gmelin, 1789), Coxim (4) (Bequaert, 1957).

9. Ornithoctona erythrocephala (Leach, 1817). Elanus leucurus (Vieillot, 1818), "Rio Paraná" (BEQUAERT, 1954).

10. Pseudolynchia brunnea (Latreille, 1812). Hydropsalis albicollis (Gmelin, 1789), Miranda (19) (BEQUAERT, 1955).

11. Pseudolynchia canariensis (Macquart, 1839). Hospedeiro não determinado, Campo Grande (21) (ZUFMS).

NYCTERIBIIDAE

12. Basilia bequaerti Guimarães \& d'Andretta, 1956. Eptesicus brasiliensis (Desmarest, 1819), Rio Brilhante (32); Nova Alvorada do Sul (28); Sidrolândia (26) (Graciolli et al., 2006). Eptesicus furinalis (d'Orbigny, 1847), Aquidauana (18) (Uniderp/Anhanguera).

13. Basilia carteri Scott, 1936. Molossus molossus (Pallas, 1766) e Platyrrhinus lineatus (E. Geoffroy, 1810), Aquidauana (9) (UNIDERP/Anhanguera). Myotis albescens (E. Geoffroy, 1806), Aquidauana (9)
(Uniderp/Anhanguera), Campo Grande (22) (Uniderp/ Anhanguera) e Corumbá (13) (Uniderp/Anhanguera, ZUFMS). Myotis nigricans (Schinz, 1821), Aquidauana (9) (UNIDERP/Anhanguera) (12) (ZUFMS, CARVALHO, 2007), Bodoquena (23) (Uniderp/Anhanguera), Campo Grande (22) (Uniderp/Anhanguera) e Corumbá (5) (Uniderp/Anhanguera), $(6,13)$ (ZUFMS). Myotis riparius Handley, 1960, Corumbá (5) (Uniderp/ Anhanguera).

14. Basilia speiseri (Miranda Ribeiro, 1907). Myotis albescens, Aquidauana $(9,11)$ (Uniderp/Anhanguera), Corumbá (6) (ZUFMS). Myotis nigricans, Aquidauana (9) (Uniderp/Anhanguera); Corumbá (5) (Uniderp/ Anhanguera), (6) (ZUFMS, CarvalHo 2007), 13 (ZUFMS); Miranda (15) (Uniderp/Anhanguera). Myotis simus Thomas, 1901, Corumbá (13) (ZUFMS). Platyrrhinus lineatus, Corumbá (13) (ZUFMS).

\section{STREBLIDAE}

\section{Streblinae}

15. Metelasmus pseudopterus Coquillett, 1907. Artibeus planirostris Spix, 1823, Jardim (29) (ZUFMS, ERIKSSON et al., 2011).

16. Strebla chrotopteri Wenzel, 1976. Chrotopterus auritus (Peters, 1856), Jardim (29) (ZUFMS, ERIKSSON et al., 2011).

17. Strebla curvata Wenzel, 1976. Glossophaga soricina (Pallas, 1766), Bodoquena (24) (ZUFMS), Jardim (29) (ERIKSSON et al., 2011).

18. Strebla diaemi Wenzel, 1966. Diaemus youngi (Jentink, 1893), Aquidauana (9) (Uniderp/Anhanguera).

19. Strebla guajiro (García \& Casal, 1965). Carollia perspicillata (L., 1758), Bodoquena (24, 25) (ZUFMS), Corumbá (7) (ZUFMS); Jardim (29) (ZUFMS, ERIKSsOn et al., 2011). Glossophaga soricina, Corumbá (14) (ZUFMS).

20. Strebla hertigi Wenzel, 1966. Phyllostomus discolor Wagner, 1843, Corumbá $(6,13)$ (CARvalho, 2007; ZUFMS).

21. Strebla mirabilis (Waterhouse, 1879). Lophostoma silvicolum d'Orbygny, 1836, Corumbá (14) (ZUFMS).

22. Strebla tonatie (Kessel, 1924). Lophostoma brasiliense Peters, 1866, Corumbá (13) (ZUFMS).

23. Strebla wiedemanni Kolenati, 1856. Desmodus rotundus (E. Geoffroy, 1810), Aquidauana (11) (Uniderp/Anhanguera), (12) (ZUFMS, CARVALHO, 2007), Corumbá $(6,7,13,14)$ (CARvalho, 2007, ZUFMS), Jardim (29) (ZUFMS, ERIKSSON et al., 2011). Glossophaga soricina, Jardim (29) (ZUFMS). Lophostoma silvicolum, Corumbá (13) (ZUFMS). Platyrrhinus lineatus, Corumbá (7) (ZUFMS). Sturnira lilium (E. Geoffroy, 1810), Aquidauana (12) (CARVAlHo, 2007).

Trichobiinae

24. Aspidoptera falcata Wenzel, 1976. Artibeus planirostris (Spix, 1823), Aquidauana (12) (CARVALHO, 2007). Carollia perspicillata (Linnaeus, 1758), Jardim (29) 
(ZUFMS, ERIKSSON et al., 2011). Desmodus rotundus (E. Geoffroy, 1810), Aquidauana (12) (CARvalHo, 2007). Glossophaga soricina (Pallas, 1766), Jardim (29) (ZUFMS, ERIKSSON et al., 2011). Platyrrhinus lineatus, Aquidauana (12) (CARvalho, 2007). Sturnira lilium (E. Geoffroy, 1810), Aquidauana (9) (Uniderp/Anhanguera), (12) (CARVALHO, 2007; ZUFMS), Bodoquena (20, 23) (Uniderp/Anhanguera), Corumbá $(6,13)$ (ZUFMS, CARVALHO, 2007), Itaporã (33) (ZUFMS, GRACIOLli et al., 2006), Jardim (29) (ZUFMS, ERIKSSON et al., 2011); Sidrolândia (30) (GRACIOLli et al., 2006).

25. Aspidoptera phyllostomatis (Perty, 1833). Artibeus lituratus (Olfers, 1818), Aquidauana (12) (CARVALHO, 2007), Corumbá (6, 13) (ZUFMS, CARvalho, 2007). Artibeus planirostris, Aquidauana (8, 9, 10, 18) (Uniderp/Anhanguera), (12) (ZUFMS, CARVALHO, 2007), Bodoquena (23) (Uniderp/Anhanguera), Bonito (27) (Graciolli et al., 2006), Corguinho (16) (Uniderp/ Anhanguera), Corumbá (5) (Uniderp/Anhanguera), (6, 13, 14) (ZUFMS, Carvalho, 2007), Jardim (29) (ZUFMS, ERIKSSON et al., 2011), Nova Alvorada do Sul (28) (GRACIOLLI et al., 2006). Carollia perspicillata, Jardim (29) (ERIKSSON et al., 2011). Desmodus rotundus, Jardim (29) (ZUFMS, ERIKSSON et al. 2011). Diaemus youngi, Corumbá (5) (Uniderp/Anhanguera). Platyrrhinus lineatus, Corumbá (1) (ZUFMS). Sturnira lilium, Aquidauana (12) (CARvalHo, 2007).

26. Exastinion clovisi (Pessôa \& Guimarães, 1937). Anoura geoffroyi Gray, 1838, Aquidauana (18) (Uniderp/Anhanguera).

27. Mastoptera minuta (Costa Lima, 1921). Artibeus planirostris, Corumbá (14) (ZUFMS). Glossophaga soricina, Corumbá (14) (ZUFMS). Lophostoma brasiliense Peters, 1866, Aquidauana (12) (CARvalHo, 2007), Corumbá(13) (ZUFMS). Lophostoma silvicolum, Aquidauana (9) (Uniderp/Anhanguera), (12) (ZUFMS, CARvalHo, 2007), Corumbá (3) (Uniderp/Anhanguera), $(6,13,14)$ (ZUFMS, Carvalho, 2007); Porto Murtinho (34) (ZUFMS). Phyllostomus hastatus (Pallas, 1767), Corumbá (6) (CARvalho, 2007). Platyrrhinus helleri (Peters, 1866), Corumbá (13) (ZUFMS).

28. Megistopoda aranea (Coquillett, 1899). Artibeus lituratus, Aquidauana (12) (CARVALHO, 2007), Corumbá $(6,7)$ (ZUFMS, CARVAlHO, 2007). Artibeus planirostris, Aquidauana $(9,11,18)$ (Uniderp/Anhanguera), (12) (ZUFMS, Carvalho, 2007), Bodoquena (23) (Uniderp/Anhanguera), Bonito (27) (Graciolli et al., 2006), Campo Grande (21) (ZUFMS), Corguinho (16) (Uniderp/Anhanguera), Corumbá $(1,6,7,13,14)$ (ZUFMS, CARVALHO, 2007), Itaporã (33) (GRACiOLli et al., 2006), Jardim (29) (ZUFMS, ERIKSSON et al., 2011), Nova Alvorada do Sul (28) (GRACIOLLi et al., 2006), Porto Murtinho (34) (ZUFMS). Carollia perspicillata, Corumbá (7) (ZUFMS), Jardim (29) (ZUFMS, ERIKSSON et al., 2011). Diaemus youngi, Corumbá (5) (Uniderp/Anhanguera). Glossophaga soricina,
Corumbá (14) (ZUFMS). Myotis riparius, Corumbá (5) (Uniderp/Anhanguera). Phyllostomus hastatus, Miranda (17) (Uniderp/Anhanguera). Platyrrhinus lineatus, Aquidauana (12) (CARvalHo, 2007), Corumbá (1) (ZUFMS). Sturnira lilium, Corumbá (7) (ZUFMS), Douradina (35) (GRACIOLLI et al., 2006).

29. Megistopoda proxima (Séguy, 1926). Artibeus planirostris, Aquidauana (12) (CARvalHo, 2007). Carollia perspicillata, Jardim (29) (ZUFMS, ERIKSSON et al., 2011). Desmodus rotundus, Aquidauana (12) (CARVAlHo, 2007). Glossophaga soricina, Corumbá (7) (ZUFMS), Jardim (29) (ZUFMS, ERIKSSON et al., 2011). Sturnira lilium, Aquidauana (9) (Uniderp/Anhanguera), (12) (CARVAlho, 2007), Bonito (27) (Graciolli et al., 2006), Corumbá (6, 7, 13) (CARVAlHo, 2007; ZUFMS), Douradina (35) (ZUFMS), Jardim (29) (ZUFMS, ERIKSSON et al., 2011), Sidrolândia $(26,30)$ (ZUFMS, Graciolli et al., 2006).

30. Noctiliostrebla aitkeni Wenzel, 1966. Noctilio albiventris Desmarest, 1818, Aquidauana (9) (Uniderp/ Anhanguera). Noctilio leporinus (L., 1758), Aquidauana (11) (Uniderp/Anhanguera).

31. Noctiliostrebla dubia (Rudow, 1871). Noctilio leporinus, Aquidauana $(9,11)$ (Uniderp/Anhanguera).

32. Noctiliostrebla maai Wenzel, 1966. Noctilio albiventris, Aquidauana (9) (Uniderp/Anhanguera), (12) (ZUFMS, Carvalho, 2007); Corumbá (6, 7, 13, 14) (CARvalho, 2007; ZUFMS), Miranda (15) (Uniderp/Anhanguera). Noctilio leporinus, Aquidauana (9) (Uniderp/Anhanguera); Corumbá (13) (ZUFMS). Platyrrhinus lineatus, Corumbá (1) (ZUFMS).

33. Paradyschiria parvula Falcoz, 1931. Artibeus planirostris, Corumbá $(13,14)$ (ZUFMS). Eumops auripendulus (Shaw, 1800), Corumbá (5) (Uniderp/ Anhanguera). Lophostoma silvicolum, Aquidauana (9) (Uniderp/Anhanguera). Noctilio albiventris, Aquidauana (9) (Uniderp/Anhanguera), (12) (ZUFMS, CARvalho, 2007); Corumbá $(1,6,7,13,14)$ (ZUFMS, Carvalho, 2007). Noctilio leporinus, Aquidauana (9) (Uniderp/Anhanguera); Corumbá (13) (ZUFMS). Phyllostomus hastatus, Aquidauana (9) (Uniderp/ Anhanguera). Platyrrhinus lineatus, Corumbá (1) (ZUFMS).

34. Paratrichobius longicrus (Miranda Ribeiro, 1907). Artibeus lituratus, Bodoquena (23) (Uniderp/ Anhanguera); Campo Grande (21) (ZUFMS). Platyrrhinus lineatus, Bonito (27) (Gracioll et al., 2006), Campo Grande (21) (ZUFMS), Jardim (29) (ZUFMS), Miranda (17) (Uniderp/Anhanguera).

35. Pseudostrebla greenwelli Wenzel, 1966. Hospedeiro não determinado, Aquidauana (12) (ZUFMS).

36. Pseudostrebla ribeiroi Costa Lima, 1921. Lophostoma silvicolum, Aquidauana (9) (Uniderp/Anhanguera), (12) (ZUFMS), Corumbá $(6,13)$ (ZUFMS, CARVALHO, 2007); Porto Murtinho (34) (ZUFMS).

37. Speiseria ambigua Kessel, 1925. Carollia perspicillata, Corumbá (7) (ZUFMS), Jardim (29) (ZUFMS, 
ERIKSSON et al., 2011). Glossophaga soricina, Corumbá (6) (ZUFMS); Jardim (29) (ZUFMS, ERIKSSON et al., 2011).

38. Trichobioides perspicillatus (Pessôa \& Galvão, 1936). Phyllostomus discolor, Aquidauana (9) (Uniderp/ Anhanguera), (12) (ZUFMS, Carvalho, 2007), Corumbá $(6,13,14)$ (ZUFMS, Carvalho, 2007); Miranda (17) (Uniderp/Anhanguera). Phyllostomus elongatus (E. Geoffroy, 1810), Corumbá (13) (ZUFMS).

39. Trichobius affinis Wenzel, 1976. Lophostoma brasiliense, Aquidauana (12) (CARVALHO, 2007).

40. Trichobius angulatus Wenzel, 1976. Artibeus planirostris, Corumbá (13) (ZUFMS). Glossophaga soricina, Corumbá (6) (CARVAlHo, 2007), Jardim (29) (ZUFMS). Platyrrhinus lineatus, Aquidauana (12) (ZUFMS), Bodoquena (23) (Uniderp/Anhanguera); Corumbá (13) (ZUFMS); Jardim (29) (ZUFMS, ERIKSSON et al., 2011).

41. Trichobius costalimai Guimarães, 1938. Phyllostomus discolor, Aquidauana $(9,11)$ (Uniderp/Anhanguera), (12) (CARvalho, 2007); Corumbá $(6,13,14)$ (ZUFMS, CARVAlHo, 2007); Miranda (17) (Uniderp/Anhanguera). Phyllostomus elongatus, Corumbá (13) (ZUFMS).

42. Trichobius diaemi Wenzel, 1976. Diaemus youngi, Aquidauana, (9) (Uniderp/Anhanguera). Myotis albescens, Corumbá (5) (Uniderp/Anhanguera).

43. Trichobius dugesii Townsend, 1891. Diaemus youngi, Aquidauana (12) (CARvalho, 2007). Glossophaga soricina, Aquidauana (12); Corumbá (6) (CARvalHo, 2007); Jardim (29) (ZUFMS, ERIKSSON et al., 2011).

44. Trichobius galei Wenzel, 1966. Micronycteris sanborni (Simmons, 1996), Corumbá (14) (ZUFMS). Natalus macrourus (Gervais, 1856), Corumbá (14) (ZUFMS).

45. Trichobius joblingi Wenzel, 1966. Anoura caudifer (E. Geoffroy, 1818), Jardim (29) (ERIKSSON et al., 2011). Artibeus planirostris, Corumbá (7) (ZUFMS), Jardim (29) (ZUFMS, ERIKSSON et al., 2011). Carollia perspicillata, Aquidauana (12) (CARVALHO 2007), (18) (Uniderp/Anhanguera); Bodoquena (23) (Uniderp/Anhanguera), (24, 25) (ZUFMS); Bonito (27) (Graciolli et al., 2006); Campo Grande (21) (ZUFMS); Corumbá (7, 13, 14) (ZUFMS); Jardim (29) (ZUFMS, ERIKSSON et al., 2011); Nova Alvorada do Sul (28) (GRACIOLLi et al., 2006, ZUFMS). Glossophaga soricina, Aquidauana (9) (Uniderp/Anhanguera), (12) (CARvalho, 2007); Corumbá $(6,7,14)$ (ZUFMS); Porto Murtinho (34) (ZUFMS). Lophostoma silvicolum, Corumbá (14) (ZUFMS). Mimon bennettii (Gray, 1838), Aquidauana (18) (Uniderp/Anhanguera). Platyrrhinus lineatus, Aquidauana (12) (CARvalHo, 2007); Campo Grande (21) (ZUFMS); Jardim (29) (ZUFMS). Sturnira lilium, Jardim (29) (ZUFMS, ERIKSSON et al., 2011). Tonatia bidens (Spix, 1823), Aquidauana (18) (Uniderp/ Anhanguera).

46. Trichobius johnsonae Wenzel, 1966. Natalus macrourus, Bodoquena (24) (ZUFMS).

47. Trichobius longipes (Rudow, 1871). Phyllostomus discolor, Corumbá (5) (Uniderp/Anhanguera). Phyllostomus hastatus, Aquidauana $(9,11,18)$ (Uniderp/ Anhanguera), (12) (CARvalHo, 2007); Bodoquena (25) (ZUFMS); Corumbá (13) (ZUFMS), Miranda (17) (Uniderp/Anhanguera). Promops centralis Thomas, 1915, Corumbá (5) (Uniderp/Anhanguera).

48. Trichobius parasiticus Gervais, 1844. Chrotopterus auritus, Aquidauana (12) (CARvalHo, 2007). Desmodus rotundus, Aquidauana (11) (Uniderp/Anhanguera), (12) (CARvalho, 2007). Corumbá $(13,14)$ (ZUFMS). Platyrrhinus helleri, Corumbá (13) (ZUFMS).

49. Trichobius persimilis Wenzel, 1976. Sturnira lilium, Aquidauana (12) (CARvalHo, 2007).

50. Trichobius silvicolae Wenzel, 1976. Lophostoma brasiliense, Corumbá (13) (ZUFMS). Lophostoma silvicolum, Aquidauana (9) (Uniderp/Anhanguera), (12) (CARvalho, 2007); Corumbá (3) (Uniderp/Anhanguera), $(6,13,14)$ (CARvalho, 2007). Phyllostomus hastatus, Corumbá (6) (CARvalHo, 2007).

51. Trichobius tiptoni Wenzel, 1976. Anoura caudifer, Bodoquena (25) (ZUFMS); Jardim (29) (ZUFMS).

52. Trichobius uniformis Curran, 1935. Artibeus planirostris, Corumbá (6) (ZUFMS). Glossophaga soricina, Aquidauana (9) (Uniderp/Anhanguera), (12) (CARVAlHo, 2007); Bodoquena (24) (ZUFMS); Corumbá (14) (ZUFMS); Jardim (29) (ZUFMS, ERIKSSON et al., 2011). Sturnira lilium, Jardim (29) (ERIKSSON et al., 2011).

53. Xenotrichobius noctilionis Wenzel, 1976. Noctilio albiventris, Corumbá (13) (ZUFMS).

Comentários sobre a lista, riqueza do estado comparado com outras regiões. Os estados com maior de número de espécies de Hippoboscoidea são Paraná e São Paulo com 57 e 56 espécies registradas, respectivamente. $\mathrm{O}$ Mato Grosso do Sul é o terceiro estado (53); no entanto o número de espécies de Nycteribiidae é somente três, número inferior a outras unidades da Federação como Santa Catarina, onde há oito espécies registradas. Esta informação evidencia que o conhecimento sobre a distribuição geográfica das três famílias de Hippoboscoidea nos estados e regiões do Brasil não é uniforme, sendo normalmente o número de Hippoboscidae inferior ao de Nycteribiidae e Streblidae.

Um dos fatores que pode estar influenciando esta diferença é que hipoboscídeos parasitam aves e cervídeos, enquanto nicteribíideos e estreblídeos são encontrados em morcegos. Pela facilidade de captura e de manipulação do hospedeiro e a melhor visualização dos dípteros sobre o corpo de morcegos, vários artigos têm sido publicados sobre a ocorrência de dípteros ectoparasitos de morcegos no Brasil (por exemplo, DiAs et al., 2009; ERIKSSON et al., 2011; Graciolli \& Bianconi, 2007; Graciolli et al., 2010; LOURENÇO \& ESBÉRARD, 2011; AGUIAR \& ANTONINI, 2011). Além disso, artigos recentes sobre a taxonomia dessas famílias de dípteros ectoparasitos de morcegos têm facilitado a identificação dos gêneros e das espécies e despertado o interesse de estudantes e pesquisadores (DICK 
\& Miller, 2010; Graciolli, 2004; 2010; Graciolli \& Azevedo, 2011; Graciolli \& Carvalho, 2001a; 2001b; Graciolli \& Dick, 2012; Graciolli \& Moura, 2005), enquanto importantes contribuições sobre a taxonomia de hipoboscídeos neotropicais são mais escassos (GRACIOLLI \& Carvalho, 2003; Wood, 2010), sendo os mais importantes publicados a mais 40 anos (BEQUAERT, 1954; 1955; 1957; MAA, 1969; Peterson \& MAA, 1970), por exemplo.

Das 35 localidades onde foram coletados espécimes de Hippoboscoidea, 16 estão localizadas no Pantanal, o mesmo número no Cerrado e três na Mata Atlântica (Fig. 1).

O Pantanal é o bioma com maior número de espécies de Hippoboscoidea registradas, quatro de Hippoboscidae, duas de Nycteribiidae e 33 de Streblidae, totalizando 39 táxons. Para o Cerrado foram registradas sete espécies de Hippoboscidae, duas de Nycteribiidae e 20 de Streblidae, totalizando 29 espécies; e para a Floresta Atlântica foram encontradas uma de Nycteribiidae e três de Streblidae, perfazendo quatro espécies.

Principais lacunas de conhecimento. Embora em comparação com o restante do país Mato Grosso do Sul seja o terceiro estado com o número de espécies, o conhecimento sobre a distribuição nos biomas e os hospedeiros de Hippoboscoidea neste estado ainda é incipiente. Com exceção de algumas localidades na Serra da Bodoquena e na região de Passo do Lontra no Pantanal, os registros aqui apresentados são de coletas realizadas de forma não sistemática e ocasional. Embora seja o bioma melhor amostrado, a maior parte do Pantanal continua não amostrada, assim como, grande parte do Cerrado, especialmente a região nordeste e leste. Enquanto isso, a Floresta Atlântica possui a maior lacuna de amostragem e conhecimento (Fig. 1).

Perspectivas de pesquisa para os próximos 10 anos. Para Mato Grosso do Sul é esperada uma grande diversidade de espécies, visto que o estado possui extensas áreas de três biomas brasileiros. Por isso, espera-se um intenso aumento no conhecimento sobre as famílias de Hippoboscoidea no estado e no país, caso as seguintes medidas sejam realizadas: (a) continuação e ampliação de programas sobre biodiversidade (por exemplo, AER da Serra da Bodoquena, AER Taboco e BIOTA/MS) que incentivem a realização de inventários em regiões pouco estudadas; (b) formação de taxonomistas que produzam chaves de identificação para gêneros e espécies de fácil utilização por outros pesquisadores como ornitólogos e mastozoólogos, além de outros profissionais e (c) maior participação de ornitólogos e mastozoólogos na coleta dos ectoparasitos, pois são os pesquisadores que normalmente estão em contato com aves e mamíferos em campo e têm condições de garantir uma identificação específica confiável dos mesmos. Já seria um grande auxílio a revisão visual e a remoção dos parasitos apenas dos espécimes que serão coletados e incluídos em coleções científicas.

Agradecimentos. A Fundação de Apoio ao Desenvolvimento do Ensino, Ciências e Tecnologia do Estado de Mato Grosso do Sul (Fundect) e a Superintendência de Ciências e Tecnologia do Estado de Mato Grosso do Sul (Sucitec/MS) pelo convite de participação neste fascículo especial da Iheringia, Série Zoologia e o suporte financeiro para sua publicação.
A Fundação Ao Conselho Nacional de Desenvolvimento Científico e Tecnológico (CNPq) (Proc. 475487/2010-9) pelo auxílio conferido ao projeto "Estutura de comunidades de artrópodos ectoparasitos de morcegos no Pantanal sul-matogrossense: uma análise por modelos nulos" e pela bolsa de produtividade de GG. À CAPES pela bolsa de mestrado de CLS e de doutorado de LFACC. À FAPESP pela bolsa (Proc. 2011/16621-9) de mestrado de DMCA. A Fernando Costa Straube e Rudi Ricardo Laps pelas informações sobre localidades e hospedeiros de hipoboscídeos.

\section{REFERÊNCIAS BIBLIOGRÁFICAS}

Aguiar, L. M. DE S. \& Antonini, Y. 2011. Descriptive ecology of bat flies (Diptera: Hippoboscoidea) associated with vampire bats (Chiroptera: Phyllostomidae) in the cerrado of Central Brazil. Memórias do Instituto Oswaldo Cruz 106:170-176.

Aguiar, L. M. de S.; Camargo, W. R. \& Portella, A. De S. 2006. Occurrence of white-winged vampire bat, Diaemus youngi (Mammalia, Chiroptera), in the Cerrado of Distrito Federal, Brazil. Revista Brasileira de Zoologia 23:893-896.

BEQUAERT, J. 1954. The Hippoboscidae or louse-flies (Diptera) of mammals and birds Part II. Taxonomy, evolution and revision of American genera and species. Entomologica Americana, News Series 34:1-232.

BequaERT, J. 1955. The Hippoboscidae or louse-flies (Diptera) of mammals and birds Part II. Taxonomy, evolution and revision of American genera and species. Entomologica Americana, New Series 35:233-416.

BequaERT, J. 1957. The Hippoboscidae or louse-flies (Diptera) of mammals and birds. Part II. Taxonomy, evolution and revision of American genera and species. Entomologica Americana, New Series 36:417-611.

Carvalho, L. F. A. C. 2007. Riqueza e diversidade de dípteros ectoparasitos de morcegos no Pantanal da Nhecolândia. Dissertação de Mestrado. Campo Grande, Universidade Federal de Mato Grosso do Sul.

CBRO - Comitê Brasileiro de Registros Ornitológicos 2011. Listas das aves do Brasil. 10ed, 25/1/2011. Disponível em < http://www.cbro. org.br>. Acessado em 14/05/2012.

Dias, P. A.; Santos, C. L. C. Dos S.; Rodrigues, F. S.; Rosa, L. C.; Lobato, K. S. \& RebÊlo, J. M. M. 2009. Espécies de moscas ectoparasitas (Diptera, Hippoboscoidea) de morcegos (Mammalia, Chiroptera) no estado do Maranhão. Revista Brasileira de Entomologia 53:128-133.

Dick, C. W. \& Graciolli, G. 2008. Checklist of the World Streblidae (Diptera: Hippoboscoidea). Disponível em: $<$ http://fm1.fieldmuseum. org/aa/Files/cdick/Streblidae_Checklist_18sep08.pdf $>$. Acessado em 01/08/2012.

Dick, C. W. \& Miller, J. A. 2010. Streblidae. In: Brown, B. V.; Borkent, A.; Cumming, J. M.; Wood, D. M.; Woodley, N. E. \& Zumbado, M. A. eds. Manual of Central American Diptera. vol. 2. Ottawa, NRC Research Press, p.1249-1260.

Eriksson, A.; Graciolli, G. \& Fisher, E. 2011. Bat flies on phyllostomid hosts in the Cerrado region: component community, prevalence and intensity of parasitism. Memórias do Instituto Oswaldo Cruz 106(3):274-278.

Garbino, G. S. T. \& TEJEDOR, A. 2013. Natalus macrourus (Gervais, 1856) (Chiroptera: Natalidae) is a senior synonym of Natalus espirosantensis (Ruschi, 1951). Mammalia 77:237-240.

Gardner, A. L. 2007. Mammals of South America. Marsupials, xenartharns, shrews, and bats. vol. 1. Chicago, The University Chicago Press. 669p.

Graciolli, G. 2004. Nycteribiidae (Diptera, Hippoboscoidea) no Sul do Brasil. Revista Brasileira de Zoologia 21:971-985.

2010. Nycteribiidae. In: Brown, B. V.; Borkent, A.; Cumming, J. M.; Wood, D. M.; Woodley, N. E. \& Zumbado, M. A. eds. Manual of Central American Diptera. vol. 2. Ottawa, NRC Research Press, p.1261-1266.

Graciolli, G.; Autino, A. G. \& Claps, G. L. 2007. Catalogue of American Nycteribiidae (Diptera, Hippoboscoidea). Revista Brasileira de Entomologia 51:142-159.

Graciolli, G. \& Azevedo, A. A. 2011. Ectoparasites of bats (Chiroptera, Furipteridae), with a description of a new species of Synthesiostrebla Twonsend (Diptera, Streblidae) from Brazil. Revista Brasileira de Entomologia 55:501-504. 
Graciolli, G.; Azevedo, A. A.; Arzua, M.; Barros-Battesti, D. M. \& Linardi, P. M. 2008. Artrópodos Ectoparasitos de Morcegos no Brasil. In: Pacheco, S. M.; Marques, R. V. \& Esbérard, C. E. L. org. Morcegos no Brasil. Biologia, sistemática, ecologia e conservação. Porto Alegre, Armazém Digital, p.123-138.

Graciolli, G. \& Bianconi, G. V. 2007. Moscas ectoparasitas (Diptera, Streblidae e Nycteribiidae) em morcegos (Mammalia, Chiroptera) em área de Floresta com Araucária no estado do Paraná, sul do Brasil. Revista Brasileira de Zoologia 24:246-249.

Graciolli, G., Cárceres, N. C. \& Bornschein, M. R. 2006. Novos registros de moscas ectoparasitas (Diptera, Streblidae e Nycteribiidae) de morcegos (Mammalia, Chiroptera) em áreas de transição cerradofloresta estacional no Mato Grosso do Sul, Brasil. Biota Neotropica 6(2):1-4.

Graciolli, G. \& Carvalho, C. J. B. 2001a. Moscas ectoparasitas (Diptera, Hippoboscoidea, Nycteribiidae) de morcegos (Mammalia, Chiroptera) do Estado do Paraná, Brasil. I. Basilia, taxonomia e chave pictórica para as espécies. Revista Brasileira de Zoologia 18(Supl.1):33-49.

Graciolli, G. \& Carvalho, C. J. B. 2001b. Moscas ectoparasitas (Diptera, Hippoboscoidea) de morcegos (Mammalia, Chiroptera) do estado do Paraná. II. Streblidae: chave pictórica para gêneros e espécies. Revista Brasileira de Zoologia 18:907-960.

Graciolli, G. \& Carvalho, C. J. B. 2003. Hippoboscidae (Diptera, Hippoboscoidea) no estado do Paraná, Brasil: chaves de identificação, hospedeiros e distribuição geográfica. Revista Brasileira de Zoologia 20:667-674.

Graciolli, G. \& Dick, C. W. 2012. Description of the second species of Joblingia Dybas \& Wenzel. Systematic Parasitology 81:187-193.

Graciolli, G. \& Moura, M. O. 2005. Basilia quadrosae sp. nov. (Diptera: Nycteribiidae), member of the ferruginea group, from Southern Brazil. Zootaxa 1087:33-38.
Graciolli, G.; Zórtea, M. \& Carvalho, L. F. A. C. 2010. Bat flies (Diptera, Streblidae and Nycteribiidae) in a Cerrado area of Goiás state, Brazil. Revista Brasileira de Entomologia 54:511-514.

Graciolli, G.; Zucco, C. A.; CançAdo, P. H. D. \& Mourão, G. 2011. Parasitism rates of Lipoptena guimaraesi and a new record of Lipoptena mazamae on Ozotoceros bezoarticus from the Central Pantanal wetlands in Brazil. Revista Brasileira de Parasitologia Veterinária 20:178-180.

LourenÇO, E. C. \& Esbérard, C. E. L. 2011. Reinfestation of Streblidae ectoparasites (Diptera) in Carollia perspicillata (Linnaeus, 1758) (Chiroptera). Revista Brasileira de Parasitologia Veterinária 20:325330.

MAA, T. C. 1969. A revised checklist and concise host index of Hippoboscidae (Diptera). Pacific Insects Monographies 20:25-204.

McAlpine, J. F. 1989. Phylogeny and classification of the Muscomorpha. In: MacAlpine, J. F. \& Wood, D. M. eds. Manual of Neartic Diptera. vol. 3. Ottawa, Minister of Suply and Services. Monograph, 28, p.1397-1518.

Peterson, B. V. \& MAA, T. C. 1970. A new Lipoptena from Chile, with a key to New World species (Diptera - Hippoboscidae). The Canadian Entomologist 102:1117-1122.

Poinar, G. \& Brown, A. 2012. The first fossil streblid bat fly, Enischnomyia stegosoma n. g., n. sp. (Diptera: Hippoboscoidea: Streblidae). Systematic Parasitology 81:79-86.

Tiepolo, L. M. \& Tomas, W. M. 2011. Ordem Artiodactyla. In: ReIs, N. R. dos; Peracchi, A. L.; Pedro, W. A. \& de Lima, I. P. orgs. Mamíferos do Brasil. 2ed. Londrina, Nélio R. dos Reis, p.293-313.

Wood, M.W. 2010. Hippoboscidae. In: Brown, B. V.; Borkent, A.; Cumming, J. M.; Wood, D. M.; Woodley N. E. \& Zumbado, M. A. eds. Manual of Central American Diptera. vol. 2. Ottawa, NRC Research Press, p.1241-1248. 